\title{
Oedogoniales from Dhule and Nandurbar district, Maharashtra (India)
}

\author{
A. G. Jaiswal* \\ Department of Botany, Arts, Commerce and Science College, Navapur, Nandurbar, Maharashtra, India
}

Received: 02.04.2017
Accepted: 03.05.2017
Published: 07.05.2017
*Address for
correspondence:
A. G. Jaiswal, Arts,
Commerce and Science
College, Navapur, Nandurbar,
Maharashtra, India.
E-mail: navapuracs@gmail.
com

KEY WORDS: Navapur, Oedogoniales, Sakri, Systematic account

\begin{abstract}
During the study of systematic account Oedogoniales of Sakri and Navapur Taluka, district Dhule and Nandurbar, respectively, Maharashtra, the author collected 23 taxa belonging to three genera. Oedogonium Hirn. is a dominant genus followed by Bulbochaete Hirn. while Oedocladium Hirn. is monotypic. Out of 23 taxa, Oedogonium orientale Jao and Bulbochaete borealis Hirn. are first time recorded from India. Four taxa are new record for Maharashtra and 12 taxa were recorded second time from Maharashtra. The Oedogoniales of this region has not been studied earlier. This is the first ever attempt to explore, enumerate, and taxonomically evaluate the algal components of the area. Distribution of the taxa in India has been discussed. The quantitative availability of species is also noted.
\end{abstract}

\section{INTRODUCTION}

The good deal of literature is now available on Indian Oedogoniales - Ashtekar and Kamat (1978), Barhate and Tarar (1985), Chaturvedi and Pandey (1980), Dixit (1937), Gonzalves (1981), Gonzalves and Jain (1968; 1970), Gonzalves and Sonnad (1961), Gonzalves and Yalavigi (1956), Hirn (1900), Kamat (1963; 1965; 1967; 1972; 1974); Kant and Gupta (1998), Khan and Kukreti (1977), Prescott (1951), Randhawa (1938; 1939; 1940; 1941), Smith (1950), Somashekar (1984), etc. No information exists on the Oedogoniales of Sakri and Navapur Taluka. 23 taxa belonging to three genera were collected from different places. Out of 23 taxa, two are reported for the first time from India, five taxa are new to Maharashtra, and 12 taxa have been reported second time from Maharashtra.

\section{MATERIALS AND METHODS}

During the study of algal flora of this region, the survey of Oedogoniales was carried out from December 1990 to January 1992 and March 1998 to March 1999. The collections were made at every fortnight from all possible places. The materials were preserved in $4 \%$ formaldehyde.

The Oedogoniales were identified with the help of available publications and monograph, i.e., Ashtekar and Kamat
(1978), Dixit (1937), Gonzalves (1981), Gonzalves and Jain (1968; 1970), Gonzalves and Sonnad (1961), Gonzalves and Yalavigi (1956), Hirn (1900), Kamat (1963; 1965; 1967; 1972; 1974), Kant and Gupta (1998), Prescott (1951), Randhawa (1938; 1939; 1940; 1941), Singh (1938), Smith (1950), Somashekar (1984).

Distribution of the taxa in India has been discussed. The quantitative availability of species is also noted. For quantitative abundance, abbreviations are used as follows: C - Common; RC - Rather common; VC - Very common; R - Rare; RR - Rather rare; VVR - Very very rare; VVVR - Very very very rare.

\section{Systematic Account}

- Oedogoniales G.S.West

- Oedogoniaceae Hirn.

\section{Oedogonium Hirn}

\section{Oedogonium intermedium Hirn. var. intermedium} Hirn. form intermedium Hirn. (Plate 1 - 1)

- Habitat: Gently adhered on the submerged rock in small pond on stream bank (RC), Dusane.

- Distribution: Maharashtra - Borivali (Dixit, 1937), Kolhapur (Kamat, 1963); Uttar Pradesh - Varanasi, Gorakhpur, Mirzapur, Basti; Andhra Pradesh - Kachiguda (Gonzalves, 1981). 
Oedogonium oviforme (Lew) Hirn. var. oviforme Lew. (Plate 1 - 2)

- Habitat: On river bank (C), Nagzari Dam

- Distribution: Maharashtra - Bombay:Thana (Gonzalves and Jain, 1970).

Oedogonium robustum (West and West) Tiff. (Plate 1 - 3)

- Habitat: On submerged twig in roadside pond (C), Nizampur

- Distribution: Maharashtra - Kolhapur (Kamat, 1963), Bombay: Thana (Gonzalves and Jain, 1970).

\section{Oedogonium macharastrense Kam. (Plate 1 - 4)}

- Habitat: On muddy soil, attached to small stone (RR), Kondaibari

- Distribution: Maharashtra - Kolhapur (Kamat, 1963), Khandesh: Velhala talao (Barhate and Tarar, 1985).

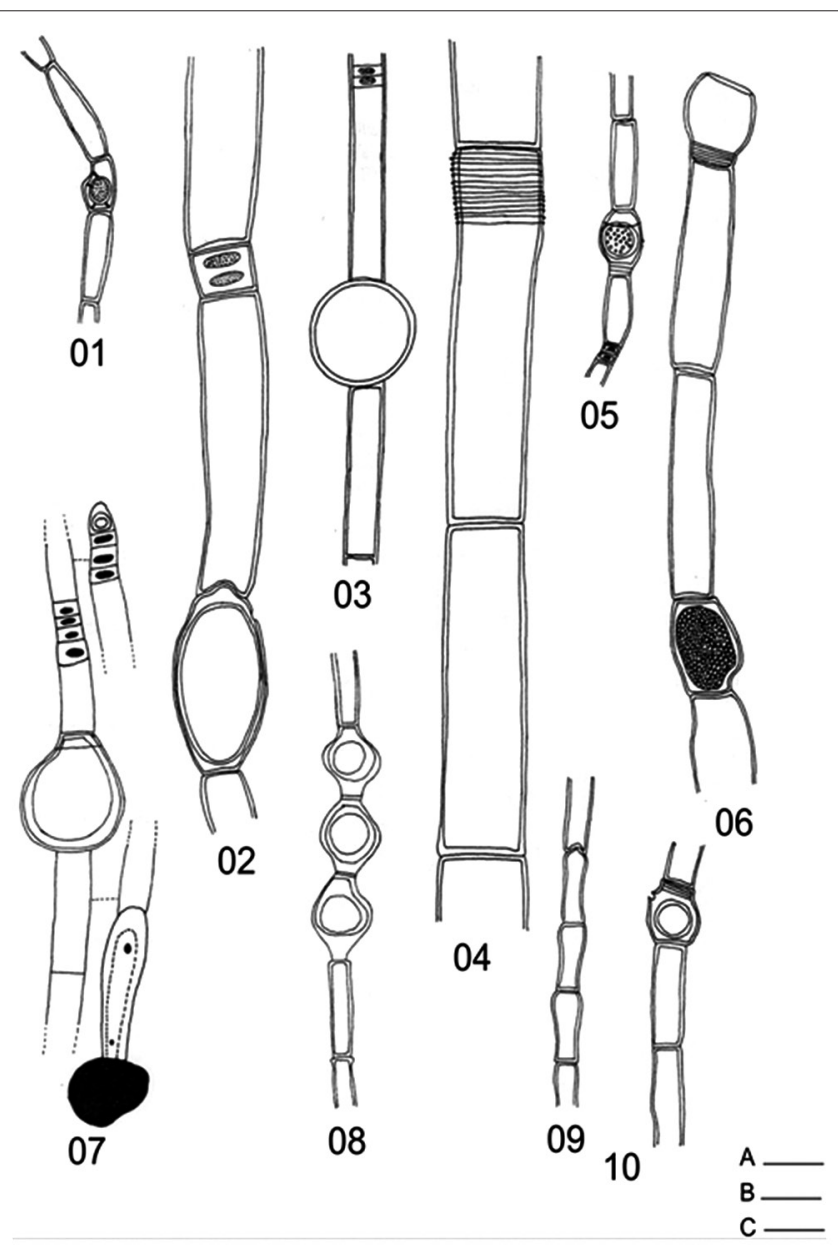

Plate 1: (Fig. 01) Oedogonium intermedium Hirn. var. intermedium Hirn. form intermedium Hirn. (Fig. 02 ) Oedogonium oviforme(Lew) Hirn. var. oviforme Lew. (Fig. 03) Oedogonium robustum (West \& West) Tiff. (Fig. 04) Oedogonium macharastrense Kam. (Fig. 05) Oedogonium mysorense Gonz. (Fig. 06) Oedogonium oblongellum Kirch ex. Hirn. var. oblongellum Hirn. (Fig. 07)Oedogonium terrestris Randhawa (Fig. 08) Oedogonium hatei Kamat (Fig. 09) Oedogonium kolhapurense Kamat (Fig. 10) Oedogonium anomalum Hirn.
Oedogonium mysorense Gonz. (Plate 1 - 5)

- Habitat: Submerged in a small pond (RC), Dahewl

- Distribution: Karnataka - Desur (Gonzalves and Sonnad, 1961).

\section{Oedogonium oblongellum Kirch ex. Hirn. var. oblongellum Hirn. (Plate 1 - 6)}

- Habitat: In a small pond (C), Cahranmal

- Distribution: Maharashtra - Bombay:Thana, Asangaon (Gonzalves and Jain, 1970), Karnataka - Naglavi (Gonzalves, 1981); Punjab - Hoshiarpur (Randhawa, 1938).

\section{Oedogonium terrestris Randhawa (Plate 1 - 7)}

- Habitat: On grass blade and on moist rock (VVVR), Kondaibari

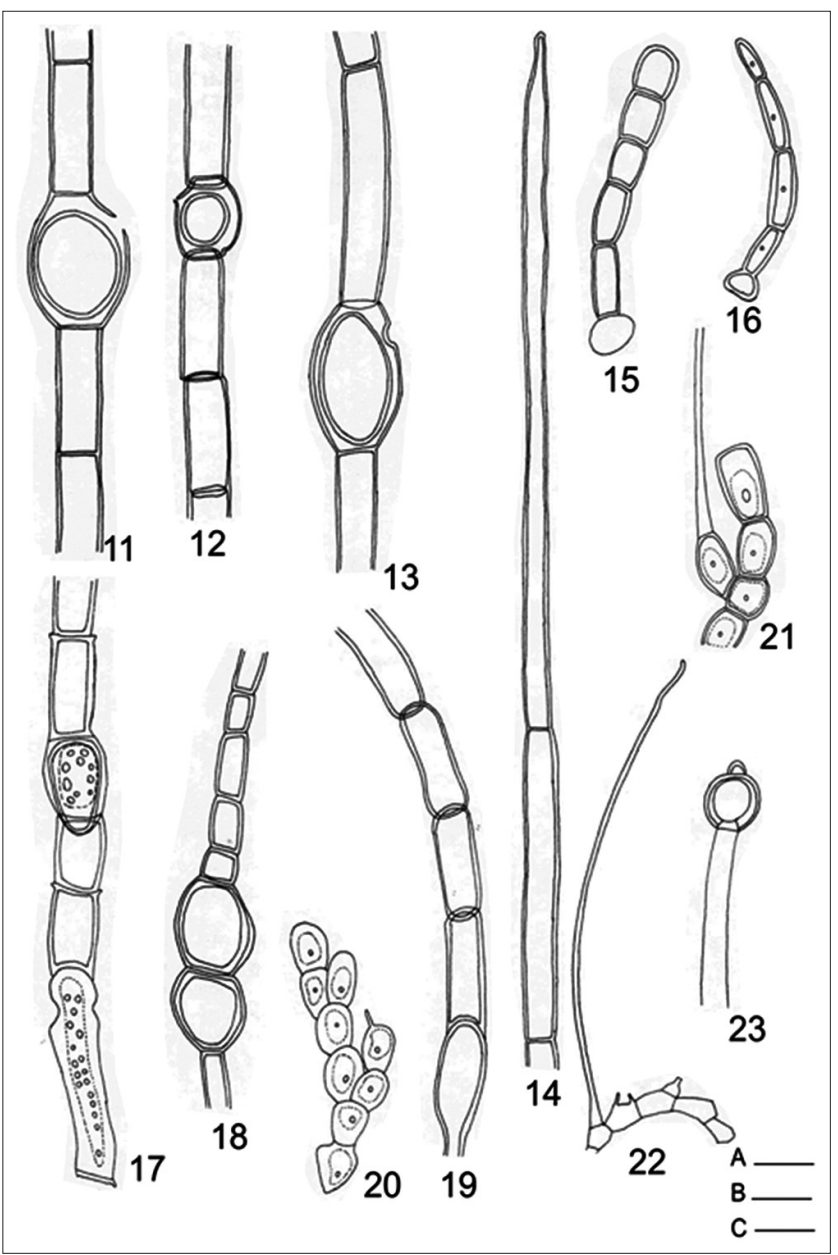

Plate 2: (Fig. 11) Oedogonium glabrum Hallas form maharastrense Kamat. (Fig. 12) Oedogonium hindustanense var. minus Gonz. \& Jain (Fig. 13) Oedogonium sociale Hirn form kanwaense Singh (Fig. 14) Oedogonium orientale Jao (Fig. 15) Oedogonium welwitschii West et. West (Fig. 16) Oedogonium welwitschii West et. West var. welwitschii Tiffany form welwitschii Tiffany (Fig. 17) Oedogonium repens Kamat (Fig. 18) Oedogonium tapeinosporum Wittrock et. Hirn. (Fig. 19) Oedogonium entirecticulosporum Gonz. \& Jain (Fig. 20) Bulbochaete nana Hirn. var. nana Hirn. (Fig. 21) Bulbochaete borealis Hirn. (Fig. 22) Bulbochaete gigantean Hirn. (Fig. 23) Oedocladium operculatum Tiffany 
- Distribution: Uttar Pradesh - Fyzabad (Randhawa, 1939), Bareilly (Chaturvedi and Pandey, 1980); Karnataka - River Kapila (Somashekar, 1984).

\section{Oedogonium hatei Kamat (Plate 1 - 8)}

- Habitat: On a submerged twig, in small pond (RC), Wasdare

- Distribution: Maharashtra - Kolhapur (Kamat, 1963).

Oedogonium kolhapurense Kamat (Plate 1 - 9)

- Habitat: Stream near Gangapur (RR), Gokul Nala

- Distribution: Maharashtra - Kolhapur (Kamat, 1963).

Oedogonium anomalum Hirn. (Plate 1 - 10)

- Habitat: Attached to submerged vegetation (RC), Vesarwadi

- Distribution: Maharashtra - Neral, Alibag (Kamat, 1968).

\section{Oedogonium glabrum Hallas form maharastrense} Kamat. (Plate 2 - 11)

- Habitat: On a submerged twig (C), Chincpada

- Distribution: Maharashtra - Kolhapur (Kamat, 1963).

Oedogonium hindustanense var. minus Gonz. and Jain (Plate 2 - 12)

- Habitat: On a submerged twig in a small pond (RC), Sukwel

- Distribution: Maharashtra - Bombay:Thana (Gonzalves and Jain, 1970); Uttarakhand - Dehradun (Khan and Kukreti, 1977).

\section{Oedogonium sociale Hirn form kanwaense Singh}

(Plate 2 - 13)

- Habitat: On stream bank (RR), Ukalapani

- Distribution: Maharashtra - Bombay: Thana, Saphala (Gonzalves and Jain, 1970); Uttar Pradesh - Banaras (Singh, 1938); Uttarakhand - Dehradun (Khan and Kukreti, 1977).

\section{Oedogonium orientale Jao (Plate 2 - 14)}

- Habitat: In a small pond, on a submerged twig (R), Ukalapani

- Distribution: Not recorded.

\section{Oedogonium welwitschii West et. West (Plate 2 - 15)}

- Habitat: Attached to the floating stem of Ipomea fustulosa (C), Navagaon

- Distribution: Maharashtra - Harsool (Ashtekar and Kamat, 1978).

O. welwitschii West et. West var. welwitschii Tiffany form welwitschii Tiffany (Plate 2 - 16)

- Habitat: On river bank and in waste water passage (VC), Borkekhadi
- Distribution: Andhra Pradesh - Hyderabad; Karnataka - Dharwar (Gonzalves, 1981).

\section{Oedogonium repens Kamat (Plate 2 - 17)}

- Habitat: On a river bank, attached to submerged vegetation (C), Sukapur

- Distribution: Maharashtra - Kolhapur (Kamat, 1963).

Oedogonium tapeinosporum Wittrock et. Hirn. (Plate 2 - 18)

- Habitat: Attached to rock near stream (C), Kudashe

- Distribution: Rajasthan - Mount Abu (Kamat, 1967); Maharashtra - Kolhapur (Kamat, 1965), Alibag (Kamat, 1968), Amravati, Tiroda (Kamat, 1972), Aurangabad (Kamat, 1974), Harsool, Mhaismal Aurangabad, Chowka (Ashtekar and Kamat, 1978); Uttarkhand - Deharadun (Khan and Kuktreti, 1977); Jammu and Kashmir - Ladakh (Kant and Gupta, 1998).

\section{Oedogonium dentirecticulosporum Gonz. and Jain} (Plate 2 - 19)

- Habitat: On river bank (VC), Shrawani

- Distribution: Maharashtra - Bombay:Thana (Gonzalves and Jain, 1968).

\section{Bulbochaete Hirn.}

Bulbochaete nana Hirn. var. nana Hirn. (Plate 2 - 20)

- Habitat: On a submerged twig in a small pond. Growing with Oedogonium sp. (R) Navapur

- Distribution: Uttar Pradesh - Ballia (Gonzalves, 1981).

Bulbochaete borealis Hirn. (Plate 2 - 21)

- Habitat: On a submerged twig in a small pond. Growing with Oedogonium sp. (R) Rayangan

- Distribution: Not recorded.

\section{Bulbochaete gigantean Hirn. (Plate 2 - 22)}

- Habitat: On a submerged twig in small pond (R) Rangawali

- Distribution: Jammu and Kashmir - Ladakh (Kant and Gupta, 1998); Karnataka - Alnavar (Gonzalves, 1981).

\section{Oedocladium Hirn.}

\section{Oedocladium operculatum Tiffany (Plate 2 - 23)}

- Habitat: On moist soil of river bank (VVR), Navapur

- Distribution: Maharashtra - Bombay: Mokhada Vada (Gonzalves and Jain, 1970); Uttar Pradesh - Almora (Randhawa, 1940); Karnataka - Dharwar (Gonzalves and Yalavigi, 1956); Himalayan range - Kumaon (Randhawa, 1941).

\section{ACKNOWLEDGMENT}

Author is thankful to UGC for providing financial assistance. Current Botany • Vol 8 • 2017 


\section{REFERENCES}

Ashtekar PV, Kamat ND. Oedogoniaceae and Zygnemataceae of Aurangabad, Maharashtra. Phykos 1978;17:35-8.

BarhateVP,Tarar JL. Additions to the algal flora of Maharashtra, Chlorophyceae from Khandesh-I. Phykos 1985;24:180-3.

Chaturvedi UK, Pandey DC. On a terrestrial Oedogonium from Rohilkhand division, U.P. Phykos 1980;19:63-5.

Dixit SC. The chlorophyceae of Bombay presidency India-I. Proc Indian Acad Sci B 1937;5:16-25.

Gonzalves EA, Jain SC. Some Oedogoniacaea from Thana district. IDIB 1970;9:1-16.

Gonzalves EA, Jain SC. Some Oedogoniacaea from Tungar hills and surrounding area. Phykos 1968;7:9-22.

Gonzalves EA, Sonnad GR. The genus Oedogonium in Mysore state. Bombay Nat Hist Soc 1961;58:715-23.

Gonzalves EA,YalavigiVS. A note on occurrence of Oedocladium operculatum Tiffany in Dharwar soils. J Karnataka Univ 1956; $1: 103-4$.

Gonzalves EA. Oedogoniales. New Delhi: ICAR; 1981.

Hirn KE. Monographie und iconographic der Oedogoniaceae. Acta Soc Sci Fenn 1900;27:1-395.

Kamat ND. Algae of Alibag, Maharashtra. J. Bombay Nat His Soc 1968;65:88-104.

Kamat ND. Algae of Marathwada, Maharashtra. Phykos 1974;13:22-32.

Kamat ND. Algae of Nainital. J Bombay Nat Hist Soc 1974;70:582-6.
Kamat ND. Ecological notes on the algae of Kolhapur. J Biosci 1965;8:47-54.

Kamat ND. On Oedocladium protonema Stalh ex. Hirn from Maharashtra. Phykos 1972;10:102-5.

Kamat ND. The algae of Kolhapur, India. Hydrobiologia 1963;22:209-305.

Kamat ND. The algae of Mount Abu. Proc Rajastan Acd Sci 1967;11:49-52.

Kant S, Gupta P. Algal Flora of Ladakh. Jodhpur, India: Scientific Publishers; 1998.

Khan M, Kukreti BC. Algal Flora of Dehradun VI Oedogoniales. Phykos 1977;16:1-8.

Prescott GW. Algae of the Western great lakes area exclusive of desmids and diatoms. Bull Cranbrook Inst Sci 1951;30:1-946.

Randhawa MS. A note on aplanospores in a species of Oedogonium. Proc Indian Acad Sci B 1937;6:230-1.

Randhawa MS. Notes on three species of Oedocladium from the Himalayas. Trans Am Microsc Soc 1941;60:417-20.

Randhawa MS. Oedogonium terrestris sp. Nov. N Hedwigia 1939; 79:281-3.

Randhawa MS. Perennation in Oedocladium operculatum Tiffany. Curr Sci 1940;9:326-7.

Smith GM. The Fresh Water Algae of the United States. New York: McGraw-Hall Book Company Inc.; 1950.

Somashekar RK. Contribution to the algal flora of river Kapila, Karnataks 1. Cyanophyceae and Chlorophyceae. Phykos 1984;23:116-24. 